VICTOR HUGO MELO'

Ana Paula Machado Botelho 2

Marcelle Marie Martins Maia ${ }^{3}$

MÁrIo DIAS CORREA JÚNIOR ${ }^{4}$

JoRGE ANDRADE PINTO ${ }^{5}$

\title{
Uso de drogas ilícitas por gestantes infectadas pelo HIV
}

\author{
Illicit drug use by pregnant women infected with HIV
}

Artigo Original

\section{Palavras-chave \\ Usuários de drogas \\ Cocaína crack \\ Complicações infecciosas na gravidez Infecções por HIV}

Transmissão vertical de doença infecciosa

Keywords

Drug users

Crack cocaine

Pregnancy complications, infectious

HIV infections

Infectious disease transmission, vertical

\section{Resumo}

OBJETIVO: Determinar se o uso de drogas ilícitas aumenta a transmissão vertical do HIV, identificar os fatores de risco envolvidos na saúde materno-infantil e a prevalência do uso de drogas entre essas gestantes. MÉTODOS: Entre 845 gestantes da região metropolitana de Belo Horizonte, Minas Gerais, atendidas no serviço entre outubro de 1997 e fevereiro de 2012, 64 (7,6\%) afirmaram usar drogas ilícitas. Os casos são as gestantes HIV positivas usuárias de drogas ilícitas (n=64) e os controles as não usuárias (n=192). Para cada caso foram selecionados três controles. Consideraram-se as diferentes exposições/condições no grupo controle como: tabagismo; etilismo; uso de tabaco e álcool; idade materna; escolaridade; etnia; e estado civil. Foram investigadas também intercorrências no pré-natal, parto e puerpério, taxa de transmissão vertical e resultados neonatais. RESULTADOS: As variáveis com significância estatística na análise univariada foram: idade materna; uso de tabaco; número de consultas de pré-natal; tipo de terapia antirretroviral; forma de contágio e carga viral na época do parto. A regressão logística mostrou como significantes: idade materna (menores de 25 anos), uso de tabaco e o número de consultas de pré-natal (menos de 6). A transmissão vertical entre usuárias foi de 4,8\% (IC95\% 1,7-13,3) e, no grupo controle, 2,1\% (IC95\% 0,8-5,2), sem diferença significante. As complicações neonatais foram mais frequentes entre os recém-nascidos das gestantes usuárias, também sem diferença significante. CONCLUSÃO: $\bigcirc$ uso de drogas ilícitas na gravidez entre mulheres infectadas pelo HIV é frequente. Assim, a abordagem sobre o uso dessas drogas deve fazer parte da rotina pré-natal. Essas gestantes são mais discriminadas, o que predispõe à negação, à não procura ou ao acesso tardio ao pré-natal. Não houve diferenças entre os grupos na transmissão vertical do vírus, o que evidencia adesão ao pré-natal e ao uso dos antirretrovirais durante a gestação.

\section{Abstract}

PURPOSE: To determine if illicit drug use increases the vertical transmission of $\mathrm{HIV}$, to identify the risk factors involved in mother and child health and the prevalence of illicit drug use among these pregnant women. METHODS: Sixtyfour (7.6\%) of 845 pregnant women from the metropolitan region of Belo Horizonte, Minas Gerais, Brazil, attended in the service between October 1997 and February 2012 reported the use of illicit drugs. Cases were HIV-positive drug users (n=64) and controls were women who did not use drugs (n=192). Three controls were selected for each case. Several conditions of exposure were considered in the control group such as tobacco use, alcohol use, alcohol and tobacco use, maternal age, educational level, ethnicity, and marital status. Problems during the prenatal period, delivery and postpartum, vertical HIV transmission and neonatal outcomes were also investigated. RESULTS: Univariate analysis showed as significant variables: maternal age, tobacco use, number of prenatal care visits, antiretroviral therapy, mode of infection, and viral load at delivery. Logistic regression revealed as significant variables: maternal age (less than 25 years); tobacco use, and number of prenatal care visits (less than 6). The vertical transmission of $\mathrm{HIV}$ was $4,8 \%(95 \% \mathrm{Cl} 1.7-13.3)$ among drug users and 2,1\% $(95 \% \mathrm{Cl} 0.8-5.2)$ in the control group, with no statistically significant difference between groups. Neonatal complications were more frequent among drug users, but also with no statistically significant difference between groups. CONCLUSION: The use of illicit drug is frequent during pregnancy among HIV-infected women. The approach to illicit drug use should be routine during prenatal care visits. These women are more discriminated against and tend to deny their habits or do not seek prenatal care. There was no difference in vertical virus transmission between groups, probably indicating adherence to antiretroviral use for antiretroviral therapies during pregnancy.
Correspondêncio

Victor Hugo Melo

Departamento de Ginecologia e Obstetrício Faculdade de Medicina da Universidade Federal de Minas Gerois Avenida Alfredo Balena, 190 (EP: $30130-100$

Belo Horizonte (MG), Bros

Recebido

$30 / 09 / 2014$

Aceito com modificacões

$10 / 10 / 2014$
Grupo de Pesquisa em HIV Materno/Infantil, Faculdade de Medicina, Hospital das Clínicas da Universidade Federal de Minas Gerais - UFMG - Belo Horizonte (MG), Brasil.

IPrograma de Pós-graduação em Saúde da Mulher, Faculdade de Medicina, Universidade Federal de Minas Gerais - UFMG Belo Horizonte (MG), Brasil.

${ }^{2}$ Curso de Graduação em Medicina, Universidade Federal de Minas Gerais - UFMG - Belo Horizonte (MG), Brasil.

${ }^{3}$ Grupo de Pesquisa em HIV Materno/Infantil, Universidade Federal de Minas Gerais - UFMG - Belo Horizonte (MG), Brasi .

${ }^{4}$ Departamento de Ginecologia e Obstetrícia, Faculdade de Medicina, Universidade Federal de Minas Gerais - UFMG Belo Horizonte (MG), Brasil.

5Departamento de Pediatria, Faculdade de Medicina, Universidade Federal de Minas Gerais - UFMG - Belo Horizonte (MG), Brasil. Conflito de interesses: não há 


\section{Introdução}

O uso de drogas, lícitas e ilícitas, é altamente prevalente em todo o mundo, estando entre os 20 maiores fatores de risco para problemas de saúde identificados pela Organização Mundial de Saúde (OMS) ${ }^{1}$. Segundo dados da Organização das Nações Unidas (ONU), extraídos do Relatório Mundial sobre Drogas e Crimes de 2013, cerca de 315 milhões de pessoas com idade entre 15 e 64 anos fizeram uso de drogas ilícitas em 2011, o que corresponde a $6,9 \%$ da população adulta mundial ${ }^{2}$. Estados Unidos, Espanha e Reino Unido lideram o consumo de drogas ilícitas no mundo, de acordo com esse mesmo relatório, sendo que maconha e haxixe são utilizados por $9,7 \%$ de pessoas na Espanha e 10,6\% no Reino Unido ${ }^{2}$.

Segundo o Centro Brasileiro de Informações sobre Drogas Psicotrópicas (CEBRID), 22,8\% da população pesquisada já fez uso de drogas ilícitas durante toda a sua vida $^{3}$. No Brasil, as drogas mais consumidas em 2005 foram: maconha $(8,8 \%)$, solventes $(6,1 \%)$, benzodiazepínicos $(5,6 \%)$, orexígenos $(4,1 \%)$ e estimulantes $(3,2 \%)^{4}$.

Apesar de diversos estudos epidemiológicos mostrarem maior prevalência de uso de drogas entre homens, o consumo de drogas entre mulheres tem aumentados. É importante destacar que, entre as consumidoras de drogas, $90 \%$ estão em idade fértil (15 a 40 anos), e $30 \%$ iniciaram o uso antes dos $20 \operatorname{anos}^{6,7}$. Entretanto, é difícil estimar com maior precisão a prevalência de uso de drogas ilícitas na gestação, uma vez que as mulheres geralmente omitem essa informação. O consumo concomitante de várias drogas e a superposição de diversos fatores socioeconômicos são variáveis importantes a serem avaliadas ${ }^{6}$.

O objetivo deste artigo é apresentar um estudo caso-controle de gestantes soropositivas para o vírus da imunodeficiência humana (HIV) acompanhadas em serviço de pré-natal de referência, para determinar se o uso de drogas ilícitas pode interferir na transmissão vertical do vírus, bem como identificar os fatores de risco envolvidos na saúde materno-infantil e a prevalência do uso dessas drogas por essas gestantes.

\section{Métodos}

\section{População e desenho do estudo}

Este estudo foi conduzido com gestantes infectadas pelo HIV em acompanhamento no Pré-natal de Alto Risco do Hospital das Clínicas da Universidade Federal de Minas Gerais (HC-UFMG) em Belo Horizonte, Minas Gerais. Todas as gestantes assinaram o Termo de Consentimento Livre e Informado e receberam atendimento multidisciplinar durante o pré-natal, parto e puerpério. As informações a respeito do uso de drogas na gestação por essas pacientes foram coletadas no banco de dados padronizado do Grupo de Pesquisa em HIV/AIDS Materno Infantil da UFMG, e nos prontuários das gestantes, no período de outubro de 1997 a fevereiro de 2012. Foram investigadas: características sociais e demográficas maternas; intercorrências durante o pré-natal, parto e puerpério; taxa de transmissão vertical; e os resultados neonatais. A coorte perinatal, iniciada em 1997, foi aprovada pelo Comitê de Ética em Pesquisa da Universidade Federal de Minas Gerais (ETIC 008/97).

Os casos foram identificados entre as gestantes HIV positivas usuárias de drogas ilícitas, conforme registro em ficha de pesquisa e prontuário médico durante o pré-natal. Os controles foram selecionados na mesma coorte de gestantes HIV positivas, entre as não usuárias de drogas ilícitas, cujo parto ocorreu próximo ao das gestantes usuárias. Foi estabelecida a proporção de 3:1 (controles: casos) para o tamanho da amostra, de acordo com o ano de nascimento do recém-nascido, a fim de garantir amostra de tamanho suficiente para as diferentes exposições avaliadas.

O estudo caso-controle conduzido foi do tipo exploratório. Para o cálculo da amostra foram consideradas as diferentes exposições/condições no grupo controle, como tabagismo, etilismo, uso concomitante de tabaco e álcool, idade materna, escolaridade, etnia e estado civil.

No levantamento do banco de dados foram encontradas 64 gestantes HIV positivas que informaram serem usuárias de drogas ilícitas, com idade entre 18 e 38 anos. Para a comparação neste estudo foram selecionadas 192 gestantes soropositivas não usuárias, que também se apresentavam na faixa etária entre 18 e 38 anos. Foram excluídas as gestantes menores de 18 anos, bem como as pacientes cujo registro no banco estava incompleto ou cujos dados não foram relatados. Foi considerada apenas a primeira gestação registrada.

\section{Análise estatística}

O banco de dados foi gerado no EpiData, versão 2.1 ${ }^{8}$, e as análises foram realizadas utilizando o SPSS, versão 21. A análise estatística foi realizada em duas etapas: em primeiro lugar foi executada a análise univariada, por meio do teste do $c^{2}$ (proporções) e testes de médias e medianas (teste $t$ de Student e teste de Wilcoxon). Foram utilizadas razões de chance (odds ratio - OR) com intervalo de confiança de $95 \%$ (IC95\%) para quantificar a associação entre os fatores de risco investigados e o uso de drogas ilícitas entre as gestantes soropositivas.

As análises multivariadas foram realizadas utilizando um modelo de regressão logística na seguinte sequência: seleção preliminar das variáveis da análise univariada, com inclusão no modelo multivariado daquelas que apresentaram $\mathrm{p}<0,25$ e algumas que, embora não tenham apresentado diferenças significativas, são descritas na literatura como 
associadas ao uso de drogas ilícitas e foram selecionadas para a análise logística multivariada. A modelagem foi realizada por meio da construção de modelos completos com todas as variáveis selecionadas para a análise e o descarte sucessivo das variáveis que não alteravam, de modo significativo, as OR relativas e os intervalos de confiança. Para a construção do modelo final o nível de significância utilizado foi de 0,05 . A avaliação dos modelos foi realizada utilizando o teste de razão da verossimilhança.

\section{Resultados}

Entre outubro de 1997 e fevereiro de 2012 foram recrutadas 845 pacientes soropositivas atendidas no serviço de pré-natal de alto risco do HC-UFMG. Entre essas, 64 $(7,6 \%)$ afirmaram ter usado drogas ilícitas em algum momento durante a gestação, ao serem perguntadas durante as consultas do pré-natal. Com relação a drogas consideradas lícitas, $52,9 \%$ afirmaram fumar durante a gravidez, 30,6\% relataram uso de álcool e 18,8\% fizeram uso de ambas as substâncias. Entre as 64 gestantes que afirmaram ser usuárias, $16,7 \%$ relataram uso de cocaína e $43,3 \%$ usaram crack; $8,3 \%$ usaram cocaína e crack; $15 \%$ usaram maconha; $8,3 \%$ usaram cocaína, crack e maconha simultaneamente e $8,4 \%$ usaram outros tipos de drogas, como inalantes.

Foram pareadas gestantes soropositivas não usuárias de drogas ( $\mathrm{n}=192)$, consideradas controles, e gestantes soropositivas usuárias de drogas $(\mathrm{n}=64)$, os casos, de acordo com o ano de nascimento. As características sociais, demográficas e clínicas maternas estão apresentadas na Tabela 1 .

Algumas características sociodemográficas e em relação à infecção foram distintas entre os casos e controles. Houve maior prevalência de gestantes com idade superior a 25 anos, mas de forma mais acentuada entre as pacientes do grupo controle: $57,8 \%$ entre as usuárias e 91,1\% no grupo controle, tendo havido diferença significante entre os grupos. O mesmo foi observado em relação à escolaridade, pois se observou que a maior parte das gestantes informou ter menos de 8 anos de estudo, sendo 67,9\% entre as usuárias e $51 \%$ entre as não usuárias. No entanto, não houve diferença significante entre os grupos. Em relação ao estado civil, houve predominância de mulheres solteiras, separadas e viúvas entre as usuárias $(57,5 \%)$, e de casadas ou vivendo em união estável com seus companheiros entre os controles $(65,6 \%)$.

O uso de tabaco foi mais prevalente entre as usuárias $(52,9 \%)$ do que entre as gestantes do grupo controle $(21,8 \%)$, com diferença significante entre os grupos. Quanto ao consumo de álcool, apesar de 30,6\% das usuárias afirmarem ter feito uso na gestação, e no grupo controle 14,5\% delas relataram o uso de bebidas alcoólicas, não houve diferença significante entre os grupos.
Todas as gestantes selecionadas compareceram às consultas de pré-natal, percebendo-se maior adesão das gestantes do grupo controle: $78,9 \%$ dessas com mais de 6 consultas, comparadas a 33,3\% das usuárias. Com relação

Tabela 1. Distribuição das variáveis selecionadas para a análise univariada em uma amostra da populaçáo de gestantes HIV positivas

\begin{tabular}{|c|c|c|c|c|c|}
\hline Variáveis & $\begin{array}{l}\text { Casos }^{a} \\
\text { n (\%) }\end{array}$ & $\begin{array}{c}\text { Controles }^{a} \\
\text { n (\%) }\end{array}$ & OR & IC95\% & $\begin{array}{c}\text { Valor } \\
\mathbf{p}\end{array}$ \\
\hline \multicolumn{6}{|l|}{ Idade } \\
\hline$\geq 25$ anos & $37(57,8)$ & $175(91,1)$ & 1 & & \\
\hline$<25$ anos & $27(42,2)$ & $17(8,9)$ & 7,5 & $3,7-15,2$ & $<0,005$ \\
\hline \multicolumn{6}{|l|}{ Etnia } \\
\hline Branca & $10(25,6)$ & $42(22,7)$ & 1 & & \\
\hline Parda & $15(38,5)$ & $76(36,2)$ & 0,8 & $0,4-1,8$ & 0,6 \\
\hline Negra & $14(35,9)$ & $76(41,1)$ & 1,1 & $0,4-2,6$ & 0,9 \\
\hline \multicolumn{6}{|l|}{ Estado civil } \\
\hline Casada, união estável & $17(42,5)$ & $126(65,6)$ & 1 & & \\
\hline Solteira, viúva, separada & $23(57,5)$ & $66(34,4)$ & 0,8 & $0,2-2,8$ & 0,7 \\
\hline \multicolumn{6}{|l|}{ Escolaridade } \\
\hline$\geq 8$ anos & $18(32,1)$ & $94(49,0)$ & 1 & & \\
\hline$<8$ anos & $38(67,9)$ & $98(51,0)$ & 1,1 & $0,3-4,5$ & 0,8 \\
\hline \multicolumn{6}{|l|}{ Uso de tabaco } \\
\hline Não & $24(47,1)$ & $147(78,2)$ & 1 & & \\
\hline Sim & $27(52,9)$ & $41(21,8)$ & 5,0 & $1,4-18,6$ & 0,01 \\
\hline \multicolumn{6}{|l|}{ Uso de álcool } \\
\hline Não & $34(69,4)$ & $148(85,5)$ & 1 & & \\
\hline Sim & $15(30,6)$ & $25(14,5)$ & 1,5 & $0,3-6,3$ & 0,6 \\
\hline \multicolumn{6}{|l|}{ Consultas pré-natal } \\
\hline$\geq 6$ & $13(33,3)$ & $86(78,9)$ & 1 & & \\
\hline$<6$ & $26(66,7)$ & $23(21,1)$ & 6,3 & $2,1-18,8$ & $<0,005$ \\
\hline \multicolumn{6}{|l|}{ Parto } \\
\hline Vaginal & $18(30,0)$ & $71(37,0)$ & 1 & & \\
\hline Cesćria & $42(70,0)$ & $121(63,0)$ & 1,4 & $0,7-2,5$ & 0,3 \\
\hline \multicolumn{6}{|l|}{ TARV } \\
\hline Profilática & $24(72,7)$ & $101(52,6)$ & 1 & & \\
\hline Terapêutica & $9(27,3)$ & $91(47,4)$ & 0,4 & $0,1-0,9$ & 0,03 \\
\hline \multicolumn{6}{|l|}{ Infecções maternas } \\
\hline Não & $12(66,7)$ & $56(75,7)$ & 1 & & \\
\hline Sim & $6(33,3)$ & $18(24,3)$ & 1,5 & $0,5-4,7$ & 0,4 \\
\hline \multicolumn{6}{|l|}{ Forma de contágio } \\
\hline Parceiro HIV+ & $26(76,5)$ & $155(100,0)$ & 1 & & \\
\hline UDI & $8(23,5)$ & - & 0,14 & $0,1-0,2$ & $<0,005$ \\
\hline \multicolumn{6}{|l|}{ AZT venoso } \\
\hline Sim & $50(89,2)$ & $178(94,6)$ & 1 & & \\
\hline Não & $6(10,8)$ & $10(5,4)$ & 2,1 & $0,7-6,1$ & 0,2 \\
\hline \multicolumn{6}{|l|}{ Contagem de células CD4 } \\
\hline$\geq 350$ células $/ \mathrm{mm}^{3}$ & $17(68,0)$ & $100(75,2)$ & 1 & & \\
\hline$<350$ células $/ \mathrm{mm}^{3}$ & $8(32,0)$ & $33(24,8)$ & 1,4 & $0,5-3,6$ & 0,5 \\
\hline \multicolumn{6}{|l|}{ Carga viral próxima ao parto } \\
\hline Indetectável & $12(50,0)$ & $97(71,8)$ & 1 & & \\
\hline Defectável & $12(50,0)$ & $35(28,2)$ & 2,8 & $1,1-6,7$ & 0,02 \\
\hline
\end{tabular}

a Houve perda de informação de algumas variáveis e nem sempre se encontra o total de casos ( $n=64)$ ou controles ( $n=192)$. OR: odds ratio; IC95\%: intervalo de confiança de 95\%; TARV: terapêutica antirretroviral; UDI: usuária de droga endovenosa; AZT: zidovudina 
à via de parto, a cesárea foi mais prevalente em ambos os grupos, com frequência de $70 \%$ entre as usuárias e $63 \%$ nas pacientes do grupo controle.

O esquema profilático, considerado como o uso de terapia antirretroviral (TARV) somente durante a gestação, para evitar a transmissão vertical do vírus foi mais frequente nos dois grupos, tendo sido utilizado por $72,7 \%$ das usuárias e $52,6 \%$ dos controles. Com relação à forma de contágio pelo HIV, houve maior prevalência da transmissão sexual, por meio do contato com parceiro soropositivo. Entre as gestantes usuárias, $76,5 \%$ afirmaram ter sido infectadas por parceiro positivo, e o mesmo ocorreu em $100 \%$ das pacientes do grupo controle.

No acompanhamento clínico dessas gestantes observou-se que a contagem de células $\mathrm{CD} 4 \geq 350$ células $/ \mathrm{mm}^{3}$ foi mais frequente entre os dois grupos: 68,0 e 75,2\%, entre usuárias e grupo controle, respectivamente. A carga viral próxima ao parto foi indetectável em $50 \%$ das gestantes usuárias de drogas ilícitas, enquanto que no grupo controle foi de $71,8 \%$. O uso do zidovudina (AZT) venoso intraparto foi frequente entre as mulheres de ambos os grupos, tendo ocorrido em $89,2 \%$ das gestantes usuárias e 94,6\% entre as do grupo controle.

Para análise do binômio mãe-filho foram avaliadas algumas características dos recém-nascidos ( $\mathrm{RN}$ ), apresentadas na Tabela 2. Observa-se que a transmissão vertical do vírus ocorreu com maior frequência entre os $\mathrm{RN}$ das gestantes usuárias de drogas ilícitas (4,8\%; IC95\% 1,7-13,3), comparados aos neonatos das gestantes do grupo controle $(2,1 \%$; IC95\% 0,8-5,2), porém sem diferença significante. Prematuridade e baixo peso ao nascer apresentaram frequências semelhantes entre os grupos. Com relação às complicações neonatais, que incluíram desconforto respiratório, sepse, icterícia precoce e distúrbios metabólicos, apesar de terem ocorrido com maior frequência entre os $\mathrm{RN}$ das gestantes usuárias, a diferença não foi significante.

As variáveis para a análise multivariada foram selecionadas a partir das Tabelas 1 e 2, adotando-se como critério de seleção o valor de $\mathrm{p}<0,25$. Para a construção do modelo, as variáveis com baixa frequência e que apresentaram colinearidade foram excluídas (tipo de TARV, forma de contágio, uso de AZT venoso e carga viral materna próxima à parto). Foram selecionadas as seguintes variáveis: idade materna, etnia, estado civil, escolaridade materna, uso de tabaco e álcool e número de consultas de pré-natal.

$\mathrm{Na}$ Tabela 3 estão apresentados os fatores de risco associados ao uso de drogas, com as razões de chance relativas e o IC95\%. Os fatores de risco associados ao uso de drogas ilícitas em gestantes HIV positivas encontrados foram: ter idade inferior a 25 anos; usar tabaco; e realizar menos de 6 consultas de pré-natal.
Tabela 2. Distribuição das variáveis selecionadas para a análise univariada das características dos neonatos de mulheres HIV positivas

\begin{tabular}{|c|c|c|c|c|c|}
\hline Variáveis & $\begin{array}{l}\text { Casos }^{0} \\
\mathrm{n}(\%)\end{array}$ & $\begin{array}{c}\text { Controles }^{a} \\
\mathrm{n}(\%)\end{array}$ & OR & IC95\% & $\begin{array}{c}\text { Valor } \\
p\end{array}$ \\
\hline \multicolumn{6}{|c|}{ Transmissão vertical } \\
\hline Não & $59(95,2)$ & $188(98,0)$ & 1 & & \\
\hline Sim & $3(4,8)$ & $4(2,0)$ & 2,4 & $0,5-11,0$ & 0,3 \\
\hline \multicolumn{6}{|c|}{ Prematuridade } \\
\hline Não & $53(88,4)$ & $164(85,4)$ & 1 & & \\
\hline Sim & $7(11,6)$ & $28(14,6)$ & 0,8 & $0,3-1,9$ & 0,6 \\
\hline \multicolumn{6}{|c|}{ Baixo peso } \\
\hline Não & $49(80,3)$ & $160(83,3)$ & 1 & & \\
\hline Sim & $12(19,7)$ & $32(16,7)$ & 1,2 & $0,6-2,5$ & 0,6 \\
\hline \multicolumn{6}{|c|}{ Complicações neonatais } \\
\hline Não & $46(76,7)$ & $159(85,0)$ & 1 & & \\
\hline Sim & $14(23,3)$ & $28(15,0)$ & 1,7 & $0,8-3,5$ & 0,1 \\
\hline
\end{tabular}

aHouve perda de informação de algumas variáveis e nem sempre se encontra o total de casos ( $n=64$ ) ou controles (n=192); OR: odds ratio; IC95\%: intervalo de confiança de $95 \%$

Tabela 3. Modelo final da análise de regressão logística para os fatores de risco associados ao uso de drogas ilícitas em uma amostra de gestantes HIV positivas

\begin{tabular}{llll}
\hline Variáveis & OR & IC95\% & Valor $p$ \\
\hline Idade (<25 anos) & 9,9 & $3,0-32,3$ & $<0,001$ \\
Uso de fabaco (sim) & 7,7 & $2,7-22,4$ & $<0,001$ \\
Consultas pré-natal $(<6)$ & 6,7 & $2,4-18,9$ & $<0,001$ \\
\hline
\end{tabular}

OR: odds ratio; IC95\%: intervalo de confiança de $95 \%$.

\section{Discussão}

O uso e abuso de drogas na gestação se tornou, nas duas últimas décadas, um problema de saúde pública mundial. Vários estudos têm demonstrado desfechos perinatais e neonatais desfavoráveis em gestantes usuárias de drogas, sejam elas lícitas ou ilícitas ${ }^{6,9}$. Por sua vez, o consumo de drogas entre mulheres tem aumentado 5 . Entre as consumidoras de drogas, $90 \%$ estão em idade fértil, entre 15 e 40 anos, e $30 \%$ o fazem desde antes de 20 anos $^{6,7}$. Uma estimativa exata da prevalência do uso dessas drogas na gestação é difícil, dado o policonsumo e a superposição de fatores sociais. A ausência de estudos epidemiológicos nacionais e internacionais abordando o uso e a dependência de drogas ilícitas na gestação limita a análise de seus efeitos sobre a mãe e o RN.

Segundo Heil et al. ${ }^{10}$, o uso de drogas ilícitas tem sido associado ao aumento da prevalência de gravidez não planejada e de doenças sexualmente transmissíveis (DSTs), além da infecção pelo HIV. Entre os resultados apresentados em seu estudo, $86 \%$ das gestações em mulheres usuárias de opioides foram não desejadas. De acordo com esses autores, o abuso de drogas geralmente está associado ao consumo de múltiplos tipos de substâncias, como álcool, 
tabaco, maconha, cocaína, crack, anfetaminas, heroína e alucinógenos. Para esses autores, a identificação das usuárias é um desafio, pois o uso ou abuso de drogas na gestação pode ser subdiagnosticado devido à omissão por parte das gestantes que, prevendo uma possível repreensão e desaprovação pelo profissional de saúde, podem negar ou relatar um consumo menor da substância ${ }^{10}$.

Vários fatores de risco associados ao uso e abuso de substâncias são descritos na literatura e entre eles, algumas características sociais e demográficas, tais como: mulheres jovens, especialmente adolescentes, menores de 18 anos; solteiras; baixa escolaridade, com menos de 8 anos de estudos completos ${ }^{11}$. Pesquisa realizada nos Estados Unidos mostrou maior prevalência de consumo de drogas ilícitas entre gestantes adolescentes $(16,2 \%)$, comparadas às gestantes entre 18 e 25 anos $(7,4 \%)$ e àquelas na faixa etária de 25 a 44 anos $(1,9 \%)^{12}$. Outros fatores sociais incluem: história familiar de abuso de substâncias, parceiro usuário e comportamento sexual de risco. Alguns fatores se relacionaram ao cuidado com a saúde, mais especificamente ao pré-natal. Foram considerados de risco o início tardio do pré-natal e o grande número de faltas às consultas ${ }^{11,12}$.

São diversos os instrumentos e protocolos existentes para o rastreamento do uso de drogas lícitas e ilícitas como, por exemplo: CAGE (acrônimo referente às suas quatro perguntas: Cut down, Annoyed by criticism, Guilty e Eye-opener); NET (acrônimo para Normal drinker, Eye-opener, Tolerance); T-ACE (acrônimo para Tolerance, Annoyed by criticism, Cut down, Eye-opener); TWEAK (acrônimo para Tolerance, Worry about drinking,Eye-opener, Amnesia, K/Cut down $)^{13}$. Os dois últimos foram validados para uso durante a gestação e ambos apresentam alta sensibilidade para dependência de álcool. O mais recente é o 4P's (Parents, Partner, Past, Pregnancy), criado especificamente para identificação rápida de gestantes usuárias de álcool ou drogas ilícitas. São quatro perguntas amplas sobre o consumo, que incluem consumo dos pais, parceiro, consumo passado e na gestação ${ }^{9}$. Esse rastreamento permite melhor identificação e um acompanhamento clínico mais intenso dessas gestantes.

Em nosso estudo foram encontradas 64 gestantes HIV positivas que confirmaram serem usuárias de drogas ilícitas, o que corresponde a 7,6\% das gestantes participantes da coorte. Essas gestantes, atendidas em um serviço de pré-natal de alto risco, afirmaram ter usado drogas ilícitas em algum momento durante a gestação, ao serem perguntadas durante as visitas para o atendimento pré-natal. $\mathrm{Na}$ literatura nacional e internacional não encontramos estudos que relacionassem o uso de drogas lícitas ou ilícitas e a infecção pelo HIV. As prevalências de uso de drogas são estabelecidas na população obstétrica em geral. Chasnoff et al. ${ }^{9}$ conduziram um estudo de três anos de duração, cujo objetivo era identificar os fatores de risco para o uso de substâncias na gestação. Em um total de 7.818 gestantes,
$9 \%$ afirmaram terem feito uso de drogas ilícitas ou álcool, ou ambos, durante a gravidez. Testes de rastreamento para o uso de substâncias foram realizados em 4.865 dessas gestantes, sendo que 1.548 (32\%) apresentaram positividade no rastreamento do uso de drogas. Por sua vez, Yonkers et al. ${ }^{13}$ publicaram estudo referente a uma coorte de 2.684 gestantes, entre as quais 1.074 foram validadas e incluídas na análise. Destas, 114 gestantes $(11,0 \%)$ afirmaram ter feito uso de drogas na gestação.

$\mathrm{Na}$ série de casos estudada, a maioria das mulheres afirmou ter usado crack em algum momento da gestação e boa parte das gestantes também confirmou o uso concomitante de outras drogas, tanto lícitas como ilícitas. A estratificação mostrou as drogas mais consumidas em nosso meio: crack e maconha. Nos Estados Unidos, na coorte citada anteriormente, foi encontrada prevalência de $5 \%$ de uso de maconha, $1 \%$ de cocaína e menos de $1 \%$ de consumo de heroína entre as gestantes ${ }^{13}$. Outro estudo demonstrou prevalência de $7 \%$ de consumo de maconha entre as gestantes, antes de terem conhecimento de sua gravidez; após a confirmação do diagnóstico esse percentual caiu para $3 \%$. O mesmo aconteceu com o consumo de cocaína: 2 e 1\%, antes e depois do diagnóstico de gravidez, respectivamente 9 .

A redução de consumo de drogas durante a gravidez demonstra que esse é um momento de grande motivação para essas mulheres que, muitas vezes, modificam seu comportamento de drogadição para protegerem seus filhos. Estudo longitudinal realizado nos Estados Unidos encontrou média de abstinência relacionada com a gestação entre usuárias de drogas ilícitas de 57\%. Infelizmente, muitas dessas mulheres retomaram seu padrão de consumo de drogas no primeiro ano após o nascimento de seus filhos ${ }^{14}$.

Foi encontrado uso concomitante de tabaco na nossa coorte em 52,9\% das usuárias de drogas ilícitas. Quanto ao consumo de álcool, 30,6\% das usuárias afirmaram seu uso na gestação. Chasnoff et al. ${ }^{9}$ encontraram prevalência de uso concomitante de álcool e drogas ilícitas de $29 \%$ entre as gestantes antes do diagnóstico formal de gravidez, e um percentual de $15 \%$ após esse diagnóstico. Outros estudos apresentaram percentuais menores de uso de álcool e tabaco. No Canadá, $14 \%$ das gestantes afirmaram ter usado álcool e $14 \%$ fizeram uso de tabaco ${ }^{7}$.

Em nosso estudo, as características sociodemográficas maternas demonstraram maior prevalência de uso de drogas ilícitas entre as gestantes com idade $\geq 25$ anos, de etnia negra, solteiras, separadas ou viúvas. Em relação à escolaridade, a maior prevalência foi entre aquelas com menos de oito anos de estudo. O grupo de mulheres analisado é semelhante ao que tem sido descrito na literatura sobre usuários de drogas: jovens; em fase economicamente ativa e reprodutiva; com baixo poder aquisitivo; e baixo nível de escolaridade ${ }^{15}$. 
Entre as variáveis significativas no nosso estudo, destacamos o menor número de consultas de pré-natal entre as gestantes usuárias de drogas. Apesar de todas as gestantes selecionadas terem realizado diversas consultas de pré-natal, $66,7 \%$ das usuárias compareceram a menos de 6 consultas, comparadas a 21,1\% das gestantes do grupo controle. Existem evidências consistentes de que a assistência pré-natal rotineira previne a morbimortalidade materna e perinatal, pois permite a detecção e o tratamento oportuno de afecções, além de reduzir os fatores de risco que trazem complicações para a saúde da mulher e do $\mathrm{RN}^{16}$. Desde o ano 2000 o Ministério da Saúde define como cuidado pré-natal adequado aquele no qual ocorre a realização de seis ou mais consultas, preconizando que quanto maior o número de consultas pré-natais maior a garantia de uma gestação e parto seguros. Deve ser reforçada a importância do pré-natal para essas gestantes, pois o acompanhamento adequado permite a identificação precoce das situações de risco, prevenindo os resultados negativos na gestação e no período neonatal, como maior morbidade e mesmo a ocorrência de óbitos. A frequência e a regularidade no acompanhamento do pré-natal permitem o desenvolvimento do vínculo entre a gestante e o profissional de saúde, o que possibilita o aprofundamento da relação médico-paciente com maior troca de informações, facilitando a identificação de hábitos de vida prejudiciais à saúde ${ }^{17,18}$.

Com relação à forma de contágio pelo HIV, houve prevalência da transmissão sexual, por meio do contato com parceiro soropositivo. Por sua vez, a maioria das mulheres fez uso de TARV do tipo profilática durante a gestação, e de AZT durante o parto. No acompanhamento clínico dessas gestantes foi observado que a carga viral próxima ao parto foi indetectável na maioria delas. Foi observado em nossa coorte que, apesar da transmissão vertical do vírus entre as gestantes usuárias de drogas ilícitas ter ocorrido em $4,8 \%$ dos neonatos, e no grupo controle em $2 \%$, não houve associação significativa entre o uso de drogas e a transmissão vertical do HIV. Por outro lado, a taxa de transmissão vertical nos últimos anos, para todas as gestantes da nossa coorte, usuárias ou não de drogas ilícitas, tem sido próxima de $0 \%$.

Algumas complicações neonatais, como por exemplo, desconforto respiratório, sepse, icterícia precoce e distúrbios metabólicos se fizeram presentes em $23,3 \%$ dos RN de usuárias, e em $15,0 \%$ dos neonatos do grupo controle, mas essa diferença não foi significante. Ao mesmo tempo, houve $19,7 \%$ de RN com baixo peso entre as usuárias, e em 16,7\% nos RNs do grupo controle, também sem diferença significativa. A identificação dos efeitos específicos dessas drogas é difícil, dada a superposição de drogas e fatores sociais envolvidos. Por sua vez, as complicações clínicas apresentadas por crianças expostas na vida intrauterina a drogas, lícitas ou ilícitas, é dependente do tipo de droga, do tempo de exposição e da quantidade usada, além da presença de doenças ou condições concomitantes, do metabolismo materno e fetal, da sua excreção e de fatores genéticos. Dois terços dos RN de mães usuárias de drogas desenvolverão sinais de abstinência neonatal. As manifestações irão ocorrer entre o terceiro e o quinto dia após a última dose de droga utilizada pela mãe ${ }^{19,20}$.

É importante destacar que em nossa análise temos muitas variáveis com perdas de informação. Nosso estudo se baseou, inicialmente, em busca retrospectiva de dados referentes a fatores sociais, demográficos, da infecção pelo HIV e de assistência pré-natal, parto e puerpério de gestantes soropositivas, a partir de fichas padronizadas e de registros de prontuários. Assim, houve perdas de alguns dados devido à coleta inadequada ou registro incorreto das informações. Entre as gestantes usuárias, o número de perdas foi maior, o que evidencia a difícil obtenção de dados a respeito desse grupo. Ressalta-se, porém, que as perdas entre os dois grupos foram equivalentes, o que nos permitiu fazer inferências. As maiores perdas, por exemplo, foram encontradas na variável infecções maternas com possível repercussão perinatal, com $71,8 \%$ de perdas entre as usuárias e 61,45\% entre os controles. Devido a esse alto índice de perdas, essa variável não foi utilizada.

Observou-se em nossa coorte que não houve diferença significante entre o uso de drogas e a transmissão vertical do HIV, provavelmente pela adesão semelhante das gestantes dos dois grupos às orientações durante o pré-natal para o uso dos antirretrovirais, ao parto assistido por equipe qualificada, e ao não aleitamento. Por sua vez, algumas complicações neonatais foram mais prevalentes em RN de mães usuárias, mas essa diferença não foi significante, provavelmente pelo tamanho da amostra.

Finalmente, em nosso estudo identificamos alguns fatores de risco para o uso de substâncias ilícitas por gestantes HIV positivas: idade menor de 25 anos, uso de tabaco e menos de 6 consultas pré-natais em toda a gestação. Esses achados são condizentes com a escassa literatura sobre o assunto e devem alertar os profissionais de saúde a respeito da questão do uso de drogas ilícitas na gravidez. Assim, destacamos que deve ser prestada atenção integral à saúde da mulher durante a gestação, avaliando a situação social em que ela está inserida e, em especial, suas histórias de vida e condições socioeconômicas, além de possíveis comportamentos violentos e/ou autodestrutivos que possam ser provocados pelo uso destas substâncias. A gravidez estimula as mulheres a buscar auxílio no sistema de saúde, e os profissionais que as atendem devem utilizar essa demanda como uma oportunidade para a abordagem a respeito do uso dessas drogas. A detecção dessas gestantes, aliada à participação de profissionais qualificados, permitirá que sejam adotadas as medidas necessárias para melhorar a qualidade do cuidado na gestação, levando à redução da morbidade materna e neonatal. 
1. World Health Organization (WHO). Global health risks: mortality and burden of disease attributable to selected major risks [Internet]. Geneva: WHO; 2009 [cited 2013 Dec 10]. Available from: <http://www.who.int/healthinfo/global_burden_disease/ GlobalHealthRisks_report_full.pdf>

2. United Nations Office on Drugs and Crime (UNODC). World drug report 2013 [Internet]. New York: United Nations; 2013 [cited 2014 Jan 20]. Available from: <http://www.unodc.org/unodc/ secured/wdr/wdr2013/World_Drug_Report_2013.pdf>

3. Carlini EA, supervisor. II levantamento domiciliar sobre o uso de drogas psicotrópicas no Brasil: estudo envolvendo as 108 maiores cidades do país: 2005 [Internet]. São Paulo: CEBRID/ UNIFESP; 2006 [citado 2014 Nov 04]. Disponível em: <http:// www.200.144.91.102/sitenovo/download.aspx?cd=65>

4. Brasil. Secretaria Nacional de Políticas sobre Drogas. Relatório brasileiro sobre drogas [Internet]. Brasília: SENAD; 2009 [citado 2013 Jan 10]. Disponível em: <http://www.obid.senad.gov.br/ portais/OBID/biblioteca/documentos/Relatorios/328379.pdf>

5. Wiechelt SA. Introduction to the special issue: international perspectives on women's substance use. Subst Use Misuse. 2008;43(8-9):973-7.

6. Yamaguchi ET, Cardoso MMSC, Torres MLA, Andrade AG. Drogas de abuso e gravidez. Rev Psiquiatr Clín. 2008;35(Suppl 1):44-7.

7. Wong S, Ordean A, Kahan M. Substance use in pregnancy. J Obstet Gynaecol Can. 201 1;33(4):367-84.

8. Lauritsen JM, Brus M, Mayatt MA. EpiData, version 2. 1: an extended tool for validated entry and documentation of data. Odense: The Epidata Association; 2001.

9. Chasnoff IJ, McGourty RF, Bailey GW, Hutchins E, Lightfoot SO, Pawson LL, et al. The $4 \mathrm{P}^{\prime}$ s Plus screen for substance use in pregnancy: clinical application and outcomes. J Perinatol. 2005;25(6):368-74.

10. Heil SH, Jones HE, Arria A, Kaltenbach K, Coyle M, Fischer G, et al. Unintended pregnancy in opioid-abusing women. J Subst Abuse Treat. $2011 ; 40(2)$ :199-202.
1 1. Carroll KM, Chang G, Behr H, Clinton B, Kosten TR. Improving treatment outcome in pregnant, methadone-maintained women: results from a randomized clinical trial. Am J Addiction. 1995;4(1):56-9.

12. U.S. Department of Health and Human Services. Center for Behavioral Health Statistics and Quality. Substance Abuse and Mental Health Services Administration. Results from the 2012 National Survey on Drug Use and Health: summary of national findings, NSDUH Series H-46, HHS Publication No. (SMA) 13-4795. Rockville: U. S. Department of health and Human Services, Substance Abuse and Mental Health Services Administration; 2013.

13. Yonkers KA, Gotman N, Kershaw T, Forray A, Howell HB, Rounsaville BJ. Screening for prenatal substance use: development of the Substance Use Risk Profile-Pregnancy scale. Obstet Gynecol. 2010;1 16(4):827-33.

14. Ebrahim SH, Gfroerer J. Pregnancy-related substance use in the United States during 1996-1998. Obstet Gynecol. 2003;101 (2):374-9.

15. Seleghim MR, Marangoni SR, Marcon SS, Oliveira MLF. Vínculo familiar de usuários de crack atendidos em uma unidade de emergência psiquiátrica. Rev Latino-Am Enfermagem. 2011;19(5):1 163-70.

16. Domingues RMSM, Hartz ZMA, Dias MAB, Leal MC. Avaliação da adequação da assistência pré-natal na rede SUS do Município do Rio de Janeiro, Brasil. Cad Saúde Pública. 2012;28(3):425-37.

17. Chang G, Orav EJ, Jones JA, Buynitsky T, Gonzalez S, WilkinsHaug L. Self-reported alcohol and drug use in pregnant young women: a pilot study of associated factors and identification. J Addict Med. 2011 ; 5(3):221-6.

18. American College of Obstetricians and Gynecologists Committee on Health Care for Underserved Women. AGOG Committee Opinion No. 473: substance abuse reporting and pregnancy: the role of the obstetrician-gynecologist. Obstet Gynecol. 2011;117(1):200-1.

19. Baver CR, Langer JC, Shankaran S, Bada HS, Lester B, Wright LL, et al. Acute neonatal effects of cocaine exposure during pregnancy. Arch Pediatr Adolesc Med. 2005;159(9):824-34.

20. Šlamberová R. Drugs in pregnancy: the effects on mother and her progeny. Physiol Res. 2012;61(Suppl 1):S123-35. 